\title{
Prospects for Lorentz invariance violation searches with top pair production at the LHC and future hadron colliders
}

\author{
Aurélien Carle $^{\mathrm{a}}$, Nicolas Chanon ${ }^{\mathrm{b}}{ }_{\mathbb{D}}$, Stéphane Perriès $^{\mathrm{c}}$ \\ University of Lyon, Université Claude Bernard Lyon 1, CNRS/IN2P3, IP2I Lyon, UMR 5822, 69622 Villeurbanne, France
}

Received: 27 November 2019 / Accepted: 4 February 2020 / Published online: 15 February 2020

(C) The Author(s) 2020

\begin{abstract}
This paper investigates the impact of hypothetical Lorentz invariance violation on the $t \bar{t}$ production at the LHC and future hadron colliders. Possible deviations from Lorentz symmetry remain poorly constrained in the top quark sector. With a dedicated analysis of $t \bar{t}$ events produced at the LHC, bounds in the top sector can be improved by up to three orders of magnitude relative to the only measurement existing so far, performed at Tevatron. The sensitivity will be even further enhanced at the HL-LHC and future colliders.
\end{abstract}

\section{Introduction}

Lorentz Invariance is a fundamental symmetry of the Standard Model (SM), however it is not expected to be conserved necessarily at the high energy scale of quantum gravity (e.g. in string theory [1] or quantum loop gravity [2]) where spacetime could undergo violent fluctuations. Quantum field theories with non-commutative geometries introduce a fundamental length-scale, hence exhibiting Lorentz Invariance violation (LIV) [3]. Cosmologies with spacetime varying couplings are natural in some grand unified theories and lead to signatures of LIV [4]. Remnants from the symmetry breaking would manifest themselves at a lower energy, and constitute an appealing signature.

Such signatures are predicted within the "Standard Model Extension" (SME) [5,6], an Effective Field Theory (EFT) considering all possible Lorentz- and CPT-violating operators in the Lagrangian (CPT breaking implies Lorentz violation for local theories [7]) in a model-independent way, preserving gauge invariance, renormalizability, locality and observer causality. The SME was tested with atomic clocks,

\footnotetext{
a e-mail: a.carle@ipnl.in2p3.fr

be-mail: n.chanon@ipnl.in2p3.fr (corresponding author)

c e-mail: s.perries@ipnl.in2p3.fr
}

penning traps, matter and antimatter spectroscopy, colliders and astroparticle experiments (for a review, see [8]), and an impressive set of results was compiled [9].

At hadron colliders the quark sector can be probed. The quark sector is constrained mostly with flavour measurements from neutral meson mixing. The most recent search for LIV and CPT breaking in the b-quark sector was performed at LHCb, using changes in $B_{(s)}$ mixing with sidereal time [10]. However, within the SME, values of the EFT coefficients are species-dependent and are different for each quark flavour. In contrast to the b-quark sector, the top quark sector remains a vastly unexplored area for LIV searches, and its SME coefficients needs to be precisely measured. Furthermore, the top quark is the only quark decaying before hadronizing, providing the unique possibility to perform a search for LIV free of non-perturbative QCD effects.

Only one actual measurement was ever performed in the top quark sector, at the $\mathrm{D} \varnothing$ experiment (Tevatron). No evidence for LIV was found, with a $10 \%$ absolute uncertainty on the measured Lorentz violating (and CPT-conserving) SME coefficients [11]. This sensitivity, well below the precision obtained in the other quark sectors, calls for new measurements at present and future colliders. The LHC is a top factory, producing top quark pairs $(t \bar{t})$ at a high rate, and provides a unique opportunity for measuring precisely SME coefficients in the top sector. In this paper, we will derive the expected sensitivity to LIV using the top pair production signature.

\section{Theoretical setup}

The SME describes the interaction of Lorentz-violating "background fields" with the particles of the SM [6]. They can arise in theories like the string scenario [1], where certain fields acquire a non-zero vacuum expectation value thereby spontaneously breaking the Lorentz symmetry. 
Within the SME, the EFT Wilson coefficients are identified with such vacuum expectation values and are constant in a given inertial frame, taken by convention to be the suncentered frame [12]. The sun-centered frame can be considered as inertial in the lifetime of a physics experiment. The origin is placed at the sun center, the Z-axis directed north and parallel to the earth rotation axis, the $\mathrm{X}$-axis is pointing to the vernal equinox of year 2000 in the celestial sphere, and the Y-axis defined to complete the direct basis. The Xand $\mathrm{Y}$-axis are thus defining the equatorial plane, lying at an angle of $\approx 23^{\circ}$ relative to the ecliptic.

In this paper, we are interested in the Lorentz violating CPT-even part of the Lagrangian density modifying the top quark kinematics [13]:

$\mathcal{L} \supset \frac{1}{2} i\left(c_{L}\right)_{\mu \nu} \bar{Q}_{t} \gamma^{\mu} \overleftrightarrow{D}^{\nu} Q_{t}+\frac{1}{2} i\left(c_{R}\right)_{\mu \nu} \bar{U}_{t} \gamma^{\mu} \overleftrightarrow{D}^{\nu} U_{t}$

where $\left(c_{L}\right)_{\mu \nu}$ and $\left(c_{R}\right)_{\mu \nu}$ are $4 \times 4$ matrices containing top quark SME coefficients (constant in the sun-centered rest frame), $Q_{t}$ is the third generation left-handed quark doublet, $U_{t}$ is the right-handed charge-2/3 top singlet, and $D^{v}$ is the gauge-covariant derivative.

A laboratory frame on earth moves around the earth rotation axis, thus the matrices $c_{\mu \nu}$ are oscillating within this frame during a sidereal day. Top quark interactions with $c_{\mu \nu}$ result in a distinctive signature: the cross section for $t \bar{t}$ production is modulating with sidereal time in the frame of the experiment, thus exhibiting Lorentz violation. The first dedicated search for such signature in the top sector was performed by $\mathrm{D} \varnothing[11]$.

\section{Top pair production in the SME}

The matrix elements for $t \bar{t}$ production in the SME were calculated analytically [13] at leading order in perturbative QCD, assuming narrow-width approximation. Under the hypothesis that the parton distribution functions in the proton are not modified (which is the case if only the top quark receives nonzero SME coefficients), and since the phase space expression stays identical (neglecting second order modification of the dispersion relation), the ratio of SME over SM cross section is:

$w=\frac{\left|\mathcal{M}_{S M E}\right|^{2}}{\left|\mathcal{M}_{S M}\right|^{2}}$

with $\mathcal{M}_{S M E}$ and $\mathcal{M}_{S M}$ the matrix elements for $t \bar{t}$ production in the SME and in the SM. In the laboratory frame, the ratio is expressed as $w(t)=1+f(t)$, with:

$$
\begin{aligned}
f(t)= & \left(c_{L, \mu \nu}+c_{R, \mu \nu}\right) R_{\alpha}^{\mu}(t) R_{\beta}^{\nu}(t)\left(\frac{\delta_{p} P}{P}+\frac{\delta_{v} P}{P}\right)^{\alpha \beta} \\
& +c_{L, \mu \nu} R_{\alpha}^{\mu}(t) R_{\beta}^{\nu}(t)\left(\frac{\delta F}{F}+\frac{\delta \bar{F}}{\bar{F}}\right)^{\alpha \beta}
\end{aligned}
$$

where $\alpha$ and $\beta$ are summation over space-time indices, $P$ is the SM matrix element squared for $t \bar{t}$ production (either quark-antiquark annihilation or gluon fusion), $F$ and $\bar{F}$ are the SM matrix elements for top and antitop decay, while $\delta_{p} P$, $\delta_{v} P, \delta F, \delta \bar{F}$ are the SME modifications in the matrix element due respectively to propagator, production vertex, and in the top and antitop decay.

The rotation matrix $R(t)$ implements the change of reference frame from the laboratory frame to the sun-centered canonical frame, and depends on the sidereal time, owing to the earth rotation around its axis with an angular velocity $\Omega=7.29 \times 10^{-5} \mathrm{rad} \cdot \mathrm{s}^{-1}(\mathrm{SI})$. The boosts due to the rotation of the earth around its axis and the revolution around the sun are negligible relative to the top quark boost produced in collisions, and are not considered. In the following developments, for definiteness we will consider the rotation matrix constructed with the CMS experiment [14] as laboratory frame. CMS is located at an azimuth of approximately $\theta=101.28^{\circ}$ on the LHC ring; the latitude of the CMS interaction point is $\lambda=46.31^{\circ}$, and the longitude is $\ell=6.08^{\circ} \mathrm{E}$. Because the ATLAS experiment [15] is located at the opposite azimuth on the LHC ring, both experiments would lead to similar results in the following studies.

Samples of $t \bar{t}$ with dilepton decay $t \bar{t} \rightarrow b e^{ \pm} \nu \bar{b} \mu^{\mp} \bar{v}$ are generated at parton-level with MADGRAPH-aMC@NLO 2.6 [16] at leading order, with the pdf set NNPDF2.3 LO [17]. The ratio $w$ can be considered as an event weight, depending on the orientation of the detector and on the four-momenta of the particles produced in the collision, to be applied to simulation events generated at leading order in QCD. Each simulated event is given a weight $w$, depending on the event kinematics and on the sidereal timestamp, attributed to the event according to its event number. Real data would be corrected for the variation in instantaneous luminosity as in [11]; we instead assume here a flat luminosity profile with time. The selection criteria required on reconstructed particles are taken from [18]. Two jets are selected, arising from b-quark hadronization, with transverse momenta $p_{T}>30 \mathrm{GeV}$ and pseudorapidity $|\eta|<2.4$. Two leptons are required to have $p_{T}>20 \mathrm{GeV}$ and $|\eta|<2.4$. No requirement on missing transverse momentum is imposed, instead the selection on the invariant mass $m_{e \mu}>20 \mathrm{GeV}$ is applied to reject Drell-Yan background of $\tau$ lepton pairs with low invariant mass. The $t \bar{t}$ dilepton channel [19] provides a precision on the inclusive cross section similar to the precision of the lepton+jet channel [18], that was used in the DØ analysis [11].

\section{Anatomy of the LIV signatures in $t \bar{t}$}

The function $f(t)$ is computed in $t \bar{t}$ simulated events after selection, and includes modified parton-level acceptance and efficiency arising from LIV. In Eq. 3, we average terms 
relative to the event kinematics (that do not depend on time): $\left\langle A_{P}^{\alpha \beta}>=<\left(\frac{\delta_{p} P}{P}+\frac{\delta_{v} P}{P}\right)^{\alpha \beta}>\right.$ and $<A_{F}^{\alpha \beta}>=<$ $\left(\frac{\delta F}{F}+\frac{\delta \bar{F}}{\bar{F}}\right)^{\alpha \beta}>$. Off-diagonal elements in the matrices $A_{P}$ and $A_{F}$ are much smaller than the in-diagonal elements, and are neglected in calculating the sinusoidal functions $f(t)$.

Four benchmark scenarios of SME coefficients, taken from [11], are studied:

1. $c_{L, \mu \nu} \neq 0$ while $c_{R, \mu \nu}=0$,

2. $c_{R, \mu \nu} \neq 0$ while $c_{L, \mu \nu}=0$,

3. $d_{\mu \nu}=\left(c_{L, \mu \nu}-c_{R, \mu \nu}\right) / 2 \neq 0$ while $c_{\mu \nu}=\left(c_{L, \mu \nu}+\right.$ $\left.c_{R, \mu \nu}\right) / 2=0$.

4. $c_{\mu \nu} \neq 0$ while $d_{\mu \nu}=0$.

The matrices $c_{\mu \nu}$ ( $\mu$ or $v=T, X, Y, Z$ ) are assumed to be symmetric (the antisymmetric part can be absorbed in other SME terms in the Lagrangian) and traceless (the trace is Lorentz invariant). Coefficients of the type $c_{T T}$ impact only the total $t \bar{t}$ cross section [13] and are not considered further. There is indeed no handle to extract them in genuine $t \bar{t}$ measurement, where any observed deviation from the predicted cross section could be attributed to QCD effects. Similarly, $c_{Z Z}$ coefficients are not studied here, since by construction there is no sensitivity induced by earth rotation in the direction transverse to the equatorial plane. As a consequence, there is no sensitivity to $c_{T Z}=c_{Z T}$ coefficients either. Eventually, $c_{T X}=c_{X T}$ and $c_{T Y}=c_{Y T}$ could be measured, but the corresponding matrix elements contributing in $A_{P}$ and $A_{F}$ are found to be negligible and these coefficients are not considered further. The remaining coefficients induce a modulation of the $t \bar{t}$ cross section with time, an unambiguous signature for LIV.

The analysis will focus on the sinusoidal signals expected for $c_{X Z}=c_{Z X} \neq 0$ and $c_{Y Z}=c_{Z Y} \neq 0$ with harmonics at a period of one sidereal day; $c_{X X}=-c_{Y Y} \neq 0$ and $c_{X Y}=$ $c_{Y X} \neq 0$ with a period of half a sidereal day. Amplitudes of the $f(t)$ functions, at selected center-of-mass energies in $\mathrm{p}-\mathrm{p}$ collisions, are shown in Fig. 1. Amplitudes of $f(t)$ are found to be the same in the scenarios $c_{X Y}=c_{Y X}$ and $c_{X X}=-c_{Y Y}$ on the one hand, as well as in the scenarios $c_{X Z}=c_{Z X}$ and $c_{Y Z}=-c_{Z Y}$ on the other hand (although phases of the sinusoidal functions are different). Larger amplitudes of $f(t)$ are found in the benchmark scenarios $c_{X Y}=c_{Y X}$ and $c_{X X}=-c_{Y Y}$ : this confirms that the experiments have higher sensitivity to $c_{\mu \nu}$ components along directions purely in the equatorial plane.

It is observed on Fig 1 that the amplitude of $f(t)$ function is growing with $\sqrt{s}$. The simulation samples used in the SME weights computation were generated according to differential SM cross sections, involving a convolution of the SM matrix element and the parton distribution function. Since the energy carried by the incoming particles relies on the

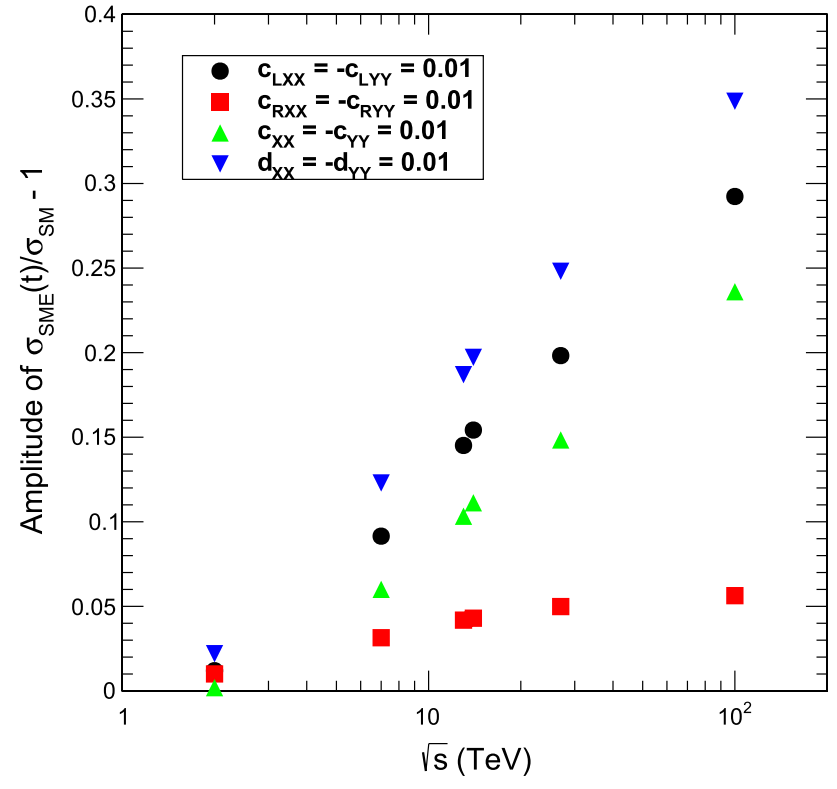

Fig. 1 Amplitude of $f(t)=\sigma_{S M E} / \sigma_{S M}-1$ in $\mathrm{p}-\mathrm{p}$ collisions at centerof-mass energies corresponding to Tevatron, LHC, HL-LHC, HE-LHC and FCC energies, using CMS location as laboratory frame, shown for the $c_{X X}=-c_{Y Y}=0.01$ benchmarks

parton distribution function at a given center-of-mass energy $\sqrt{s}$, the raising of $f(t)$ with $\sqrt{s}$ was investigated by generating dedicated samples turning off parton distribution functions in the proton, thus any remaining increase in amplitude could be attributed to the SME matrix element expression. An enhancement of the $f(t)$ amplitude as the square of the center-of-mass energy was found with these samples, compatible with the expressions for the matrix elements given in [13].

At Tevatron, $p \bar{p} \rightarrow t \bar{t}$ production was initiated mainly by $q \bar{q}$ annihilation while at the LHC, with the increase of $\sqrt{s}, g g$ fusion is dominant in $p p \rightarrow t \bar{t}$ production owing to higher gluon luminosity in the proton. We compared the $f(t)$ amplitudes obtained for $p \bar{p}$ collisions at $\mathrm{D} \varnothing$ and $p p$ collisions in the CMS laboratory frame, in samples generated at the same center-of-mass energy $\sqrt{s}=1.96 \mathrm{TeV}$, and examined separately $g g$ and $q \bar{q}$ production mechanisms. We find a larger $f(t)$ amplitude in $q \bar{q}$ than in $g g$ production mechanism. For a given $\sqrt{s}$, the experiment's position can favor one benchmark scenario or the other. Overall, the increase in center-of-mass-energy has a dominant impact on the amplitude, while the change in detector position and production mechanism induces a smaller change.

We scanned the latitude and azimuth of potential experiments on earth (the longitude does not impact the amplitude owing to the earth rotation). It was found that both ATLAS or CMS sit in a dip for the projected sensitivity on the benchmarks $c_{X X}=-c_{Y Y} \neq 0$ and $c_{X Y}=c_{Y X} \neq 0$, and in a hill on the benchmarks $c_{X Z}=c_{Z X} \neq 0$ and $c_{Y Z}=c_{Z Y} \neq 0$, 
because of the latitude and azimuth interplay with the directions of the SME coefficients [20]. In general, ATLAS and CMS sensitivity to SME coefficients will be similar since they are located at opposite azimuthal angle in the LHC ring.

\section{Sensitivity at the LHC and future colliders}

In this section, projected sensitivity at the LHC and future colliders will be studied and compared with Tevatron results. The $\mathrm{D} \emptyset$ analysis [11] at Tevatron was performed with a luminosity of $5.3 \mathrm{fb}^{-1}$ of $p-\bar{p}$ collisions at $\sqrt{s}=1.96 \mathrm{TeV}$. The LHC produces $p-p$ collisions, with about $150 \mathrm{fb}^{-1}$ of data recorded at $\sqrt{s}=13 \mathrm{TeV}$ during Run 2 [21]. The High Luminosity LHC (HL-LHC), expected to start data taking in 2027, will deliver $3 a b^{-1}$ at $\sqrt{s}=14 \mathrm{TeV}$ [22]. The High Energy LHC (HE-LHC) is a future collider option that could take place after the HL-LHC, using the same tunnel with upgraded magnets, to achieve an expected center-ofmass energy of $\sqrt{s}=27 \mathrm{TeV}$ and $15 a b^{-1}$ of integrated luminosity [23]. Eventually the Future Circular Collider, in its hadron collider stage (FCC-hh), is an even higher energy option, where a new $100 \mathrm{~km}$ tunnel nearby CERN would be built to achieve the unprecedented center-of-mass energy of $\sqrt{s}=100 \mathrm{TeV}$ and $15 a b^{-1}$ of integrated luminosity [24]. For simplicity, we assume the same coordinates as the CMS detector for an experiment at the LHC and future colliders (LHC Run 2, HL-LHC, HE-LHC, and FCC).

The SM cross section for $t \bar{t}$ production is computed at the center-of-mass energy of each collider scenario with Top++ $[25,26]$, at next-to-next-to-leading order accuracy in perturbative QCD.

The hypothesis is made that the efficiencies for selecting reconstructed particles are identical to those of [18] at the LHC and beyond. This can be regarded as optimistic if considering the increasing number of collisions piling up with the hard process at higher and higher instantaneous luminosity (from about 30 pileup events at the LHC to 1000 at the FCC). However pileup mitigation techniques have been proved to work very efficiently, and more ideas are being explored to keep pileup impact under control at the future detectors [24].

We study the $e \mu$ final state, where the background is arising mainly from single top production, and the Drell-Yan production is efficiently suppressed by requiring two leptons of different flavour. The same signal to background ratio as in [18] is also assumed (this $t \bar{t}$ channel is usually very clean with $s / b \approx 15$ ). This approximation could be refined by computing the cross section of the main backgrounds with fixed order QCD calculation, however the present value of the ratio is believed to be reasonably stable at higher centerof-mass energies.

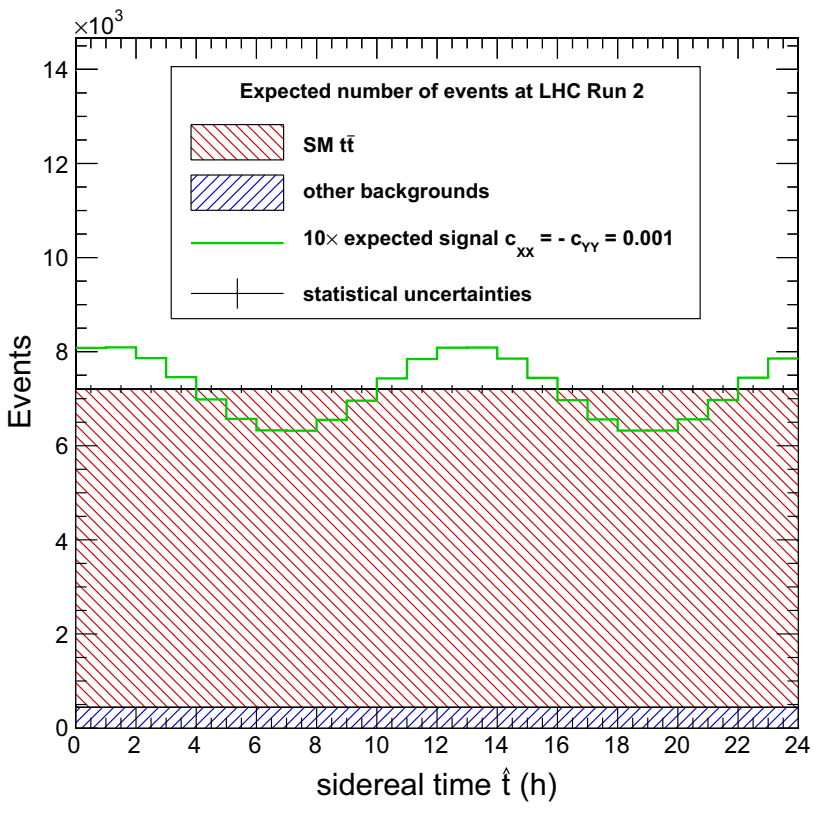

Fig. 2 Number of events expected at LHC Run 2 as a function of the sidereal time, for LIV signal in the scenario $c_{X X}=-c_{Y Y}=0.001$, SM $t \bar{t}$ and other backgrounds

The expected contributions for the LIV signal, SM $t \bar{t}$ production and single top background are fitted with a $\chi^{2}$ method to the Asimov dataset [27], using bins of one sidereal hour. The Asimov dataset represents fake data constructed from the sum of all contributions excluding the signal. The $\chi^{2}$ is constructed from the difference in the event yield in the Asimov dataset and the sum of the event yields for background and SM $t \bar{t}$ processes, the latter multiplied by $\left(1+c f\left(t_{i}\right)\right)$, where $c$ is the SME coefficient and $f\left(t_{i}\right)$ the function given in Eq. 3 in the time bin $t_{i}$. An illustration of the distribution used is shown in Fig. 2.

We perform the study for the above mentioned colliders and SME coefficient benchmarks. We obtain the projected precision on the SME coefficients. As a cross-check, we find sensitivity of the same order of magnitude as with $\chi^{2}$ method, when using HISTFACTORY [28] implementing the LHC teststatistics in a likelihood fit [29]. Systematic uncertainties are rounded from [18]: $2 \%$ is attributed to the luminosity, $4 \%$ on the inclusive measurement of $t \bar{t}$ production (the main systematic uncertainties arising from jet energy scale, lepton efficiencies and $t \bar{t}$ modeling), and $30 \%$ on the small single top background. Projected precision on the SME parameters is shown on Table 1. The systematic uncertainties are applied inclusively, with a flat profile in time. They are modeled as scaling up and down the number of signal or background events, or scaling up and down the luminosity. By contrast, the LIV signal depends on time: it is constrained in the fit thanks to the time-dependent shape of the signal model.

By performing the $\chi^{2}$ fit using $\mathrm{D} \varnothing$ location, the value of $A_{P}$ and $A_{F}$ matrices in [30] and the total number of 
Table 1 Comparison of expected precision in the measurement of the SME parameters, extrapolated from $t \bar{t}$ measurements [11,18], for $\mathrm{D} \emptyset$, LHC Run 2, HL-LHC, HE-LHC, FCC experiment

\begin{tabular}{llllll}
\hline & $\mathrm{D} \emptyset$ & LHC $($ Run 2) & HL-LHC & HE-LHC & FCC \\
\hline$\Delta c_{L X X}, \Delta c_{L X Y}$ & $1 \times 10^{-1}$ & $7 \times 10^{-4}$ & $2 \times 10^{-4}$ & $2 \times 10^{-5}$ & $5 \times 10^{-6}$ \\
$\Delta c_{L X Z}, \Delta c_{L Y Z}$ & $8 \times 10^{-2}$ & $3 \times 10^{-3}$ & $5 \times 10^{-4}$ & $9 \times 10^{-5}$ & $2 \times 10^{-5}$ \\
$\Delta c_{R X X}, \Delta c_{R X Y}$ & $9 \times 10^{-2}$ & $3 \times 10^{-3}$ & $5 \times 10^{-4}$ & $8 \times 10^{-5}$ & $5 \times 10^{-5}$ \\
$\Delta c_{R X Z}, \Delta c_{R Y Z}$ & $7 \times 10^{-2}$ & $1 \times 10^{-2}$ & $2 \times 10^{-3}$ & $4 \times 10^{-4}$ & $8 \times 10^{-5}$ \\
$\Delta c_{X X}, \Delta c_{X Y}$ & $7 \times 10^{-1}$ & $1 \times 10^{-3}$ & $2 \times 10^{-4}$ & $3 \times 10^{-5}$ & $9 \times 10^{-6}$ \\
$\Delta c_{X Z}, \Delta c_{Y Z}$ & $6 \times 10^{-1}$ & $4 \times 10^{-3}$ & $7 \times 10^{-4}$ & $1 \times 10^{-4}$ & $3 \times 10^{-5}$ \\
$\Delta d_{X X}, \Delta d_{X Y}$ & $1 \times 10^{-1}$ & $6 \times 10^{-4}$ & $1 \times 10^{-4}$ & $2 \times 10^{-5}$ & $8 \times 10^{-6}$ \\
$\Delta d_{X Z}, \Delta d_{Y Z}$ & $7 \times 10^{-2}$ & $2 \times 10^{-3}$ & $4 \times 10^{-4}$ & $8 \times 10^{-5}$ & $2 \times 10^{-5}$ \\
\hline
\end{tabular}

observed events quoted in [11], the absolute expected precision is found to be of the order of $10 \%$, compatible with the observed results in DØ analysis, thus validating the procedure.

The precision on the SME coefficients is expected to be improved by up to three orders of magnitude from $D \emptyset$ to the LHC Run 2, depending on the coefficients. An additional expected improvement is found at future hadron colliders, with up to two more orders of magnitude at the FCC. Overall, performing sidereal time analysis of $t \bar{t}$ production at present and future hadron colliders will greatly improve existing bounds on Lorentz-violating $c_{\mu \nu}$ coefficients for the top quark in the SME.

It has to be noted that parton distribution functions in the proton at $100 \mathrm{TeV}$ are subject to high uncertainties at large momentum transfer [31]. The expected results are also subject to other approximations relative to the performance of future detectors, the treatment of pileup, and the cross sections for top quark processes. Although we consider that the adopted approximations are reasonable, results of this phenomenology study should mainly be considered as providing an order of magnitude for the sensitivity rather than a precise and definitive answer, that will be given by future experiments.

The improvement found in the expected precision of the SME coefficients at the LHC and future colliders is explained by a combination of three factors: (1) the increase in SM $t \bar{t}$ cross sections with $\sqrt{s}$ relative to Tevatron, (2) the higher expected number of events produced in collisions with the greater volume of integrated luminosity, and (3) the increase in the SME over SM matrix elements for $t \bar{t}$ production and decay with $\sqrt{s}$, leading to an increase of the amplitude of the function $f(t)$ in Eq. 3 .

The present analysis can be refined in several ways. In addition to the $e \mu$ channel of $t \bar{t}$ decay, the same flavour dilepton channel and the lepton+jets channel could be used. Eventually, the $c_{\mu \nu}$ coefficients are modifying top quark kinematics, thus differential cross sections or multivariate analysis making use of kinematic $t \bar{t}$ observables could be used to improve sensitivity.

\section{Conclusions}

In this paper, we highlighted the physics potential of the LHC and future hadron colliders for LIV searches with $t \bar{t}$ production. Bounds on the top quark $c_{\mu \nu}$ coefficients in the SME can be improved by up to three orders of magnitude already at the LHC, and the total improvement is expected to reach five orders of magnitude at future colliders such as the FCC.

Other proposed searches in the top sector [13] are targeting CPT violation at hadron colliders, by measuring the charge asymmetry between single top and antitop events as a function of sidereal time. This search is experimentally very challenging, and would deserve dedicated sensitivity studies, that are postponed to a later paper.

Other LIV processes of interest would deserve detailed studies. The LHC is often thought of as a top factory, however the production of QCD and electroweak particles has also a very high cross section. By studying the production of QCD jets, $W^{ \pm}$and $Z$ bosons at present and future hadron colliders, poorly constrained areas of the SME could be probed at an unprecedented sensitivity.

Acknowledgements We would like to thank Alan Kostelecký for enlightening discussions.

Data Availability Statement This manuscript has associated data in a data repository. [Authors' comment: Expressions for the LIV signal are available in the references, and the $t \bar{t}$ samples are standard simulation.]

Open Access This article is licensed under a Creative Commons Attribution 4.0 International License, which permits use, sharing, adaptation, distribution and reproduction in any medium or format, as long as you give appropriate credit to the original author(s) and the source, provide a link to the Creative Commons licence, and indicate if changes were made. The images or other third party material in this article are included in the article's Creative Commons licence, unless indicated otherwise in a credit line to the material. If material is not included in the article's Creative Commons licence and your intended use is not permitted by statutory regulation or exceeds the permitted use, you will need to obtain permission directly from the copyright holder. To view a copy of this licence, visit http://creativecomm ons.org/licenses/by/4.0/.

Funded by $\mathrm{SCOAP}^{3}$. 


\section{References}

1. V.A. Kostelecky, S. Samuel, Phys. Rev. D 39, 683 (1989). https:// doi.org/10.1103/PhysRevD.39.683

2. R. Gambini, J. Pullin, Phys. Rev. D 59, 124021 (1999). https://doi. org/10.1103/PhysRevD.59.124021

3. S.M. Carroll, J.A. Harvey, V.A. Kostelecky, C.D. Lane, T. Okamoto, Phys. Rev. Lett. 87, 141601 (2001). https://doi.org/10. 1103/PhysRevLett.87.141601

4. V.A. Kostelecky, R. Lehnert, M.J. Perry, Phys. Rev. D 68, 123511 (2003). https://doi.org/10.1103/PhysRevD.68.123511

5. D. Colladay, V.A. Kostelecky, Phys. Rev. D 55, 6760 (1997). https:// doi.org/10.1103/PhysRevD.55.6760

6. D. Colladay, V.A. Kostelecky, Phys. Rev. D 58, 116002 (1998). https://doi.org/10.1103/PhysRevD.58.116002

7. O.W. Greenberg, Phys. Rev. Lett. 89, 231602 (2002). https://doi. org/10.1103/PhysRevLett.89.231602

8. S. Liberati, Class. Quantum Gravity 30, 133001 (2013). https://doi. org/10.1088/0264-9381/30/13/133001

9. V.A. Kostelecky, N. Russell, Rev. Mod. Phys. 83, 11 (2011). https:// doi.org/10.1103/RevModPhys.83.11

10. LHCb Collaboration, Phys. Rev. Lett. 116(24), 241601 (2016). https://doi.org/10.1103/PhysRevLett.116.241601

11. D0 Collaboration, Phys. Rev. Lett. 108, 261603 (2012). https://doi. org/10.1103/PhysRevLett.108.261603

12. R. Bluhm, V.A. Kostelecky, C.D. Lane, N. Russell, Phys. Rev. Lett. 88, 090801 (2002). https://doi.org/10.1103/PhysRevLett.88. 090801

13. M.S. Berger, V.A. Kostelecký, Z. Liu, Phys. Rev. D 93(3), 036005 (2016). https://doi.org/10.1103/PhysRevD.93.036005

14. CMS Collaboration, JINST 3, S08004 (2008). https://doi.org/10. 1088/1748-0221/3/08/S08004

15. ATLAS Collaboration, JINST 3, S08003 (2008). https://doi.org/ 10.1088/1748-0221/3/08/S08003

16. J. Alwall, R. Frederix, S. Frixione, V. Hirschi, F. Maltoni, O. Mattelaer, H.S. Shao, T. Stelzer, P. Torrielli, M. Zaro, JHEP 07, 079 (2014). https://doi.org/10.1007/JHEP07(2014)079
17. R.D. Ball et al., Nucl. Phys. B 867, 244 (2013). https://doi.org/10. 1016/j.nuclphysb.2012.10.003

18. CMS Collaboration, Eur. Phys. J. C 77, 172 (2017). https://doi.org/ 10.1140/epjc/s10052-017-4718-8

19. ATLAS Collaboration, Phys. Lett. B 761, 136 (2016). https://doi. org/10.1016/j.physletb.2016.08.019, https://doi.org/10.1016/j. physletb.2017.09.027. [Corrigendum: Phys. Lett.B772,879(2017)]

20. A. Carle, N. Chanon, S. Perries, arXiv:1909.01990 (2019)

21. CMS Collaboration, Phys. Rev. Lett. 122(13), 132001 (2019). https://doi.org/10.1103/PhysRevLett.122.132001

22. G. Apollinari, I. Béjar Alonso, O. Brüning, P. Fessia, M. Lamont, L. Rossi, L. Tavian, CERN Yellow Rep. Monogr 4, 1 (2017). https:// doi.org/10.23731/CYRM-2017-004

23. A. Abada et al., Eur. Phys. J. ST 228(5), 1109 (2019). https://doi. org/10.1140/epjst/e2019-900088-6

24. A. Abada et al., Eur. Phys. J. ST 228(4), 755 (2019). https://doi. org/10.1140/epjst/e2019-900087-0

25. M. Czakon, P. Fiedler, A. Mitov, Phys. Rev. Lett. 110, 252004 (2013). https://doi.org/10.1103/PhysRevLett.110.252004

26. M. Czakon, A. Mitov, Comput. Phys. Commun. 185, 2930 (2014). https://doi.org/10.1016/j.cpc.2014.06.021

27. G. Cowan, K. Cranmer, E. Gross, O. Vitells, Eur. Phys. J. C 71, 1554 (2011). https://doi.org/10.1140/epjc/s10052-011-1554-0, https:// doi.org/10.1140/epjc/s10052-013-2501-z. [Erratum: Eur. Phys. J. C73,2501(2013)]

28. K. Cranmer, G. Lewis, L. Moneta, A. Shibata, W. Verkerke, CERNOPEN-2012-016 (2012)

29. ATLAS Collaboration, CMS Collaboration, LHC Higgs Combination Group, CMS-NOTE-2011-005. ATL-PHYS-PUB-2011-11 (2011). http://cds.cern.ch/record/1379837

30. D.W. Whittington, FERMILAB-THESIS-2012-16 (2012). https:// doi.org/10.2172/1248357

31. J. Rojo, PoS DIS2016, 275 (2016). https://doi.org/10.22323/1.265. 0275 